\title{
COMMUTATIVE GROUP ALGEBRAS AND PRÜFER GROUPS
}

\author{
P. V. DANCHEV
}

\begin{abstract}
Suppose $G$ is a multiplicatively written abelian $p$-group, where $p$ is a prime, and $F$ is a field of arbitrary characteristic. The main results in this paper are that none of the Sylow $p$-group of all normalized units $S(F G)$ in the group ring $F G$ and its quotient group $S(F G) / G$ cannot be Prüfer groups. This contrasts a classical conjecture for which $S(F G) / G$ is a direct factor of a direct sum of generalized Prüfer groups whenever $F$ is a perfect field of characteristic $p$.
\end{abstract}

\section{Introduction}

Throughout the present paper, let $G$ be an arbitrary multiplicative abelian group, let $R$ be a commutative unitary ring of prime characteristic $p$ and let $K$ be a field of characteristic different from $p$. As usual, $R G$ and $K G$ are the group algebras over $R$ and $K$ respectively, $S(R G)$ is the $p$-torsion component of the group of all normalized units $V(R G)$ in $R G$ (note that $V(R G)=S(R G)$ when $G$ is a $p$-group), $S(K G)$ is the group of all normalized $p$-elements in $K G$, and $G_{p}$ is the maximal $p$-primary subgroup of $G$. Moreover, $N(R)$ denotes the Baer radical (often called nil-radical) of $R$. Given a subgroup $H$ of $G$ and a subring $L$ of $R$ containing the same identity, $I(L G ; H)$ denotes the relative augmentation ideal of $L G$ with respect to $H$. Terminology and notations follow [12], [15] and [16].

For instance, following [12] and [13], we shall say that the abelian $p$-group $A$ is a Prüfer group if $A^{p^{\omega}}$ is cyclic of order $p$ and $A / A^{p^{\omega}}=\oplus_{n<\omega} \mathrm{Z}\left(p^{n}\right)$, where $\mathrm{Z}\left(p^{n}\right)$ is a cyclic group of order $p^{n}$. Certainly, $A$ is an infinite countable group, and the non-zero Ulm-Kaplansky functions of $A / A^{p^{\omega}}$ are equal to 1 . In fact, we know that $B G^{p^{\omega}} / G^{p^{\omega}}$ is a $p$-basic subgroup of $G / G^{p^{\omega}}$ whenever $B$ is a $p$-basic subgroup of $G$. So, for the $n$-Ulm-Kaplansky functions $f_{n}$, where $n \geq 0$ is an integer, it follows that $f_{n}\left(G / G^{p^{\omega}}\right)=$ $f_{n}\left(B G^{p^{\omega}} / G^{p^{\omega}}\right.$ ) and $f_{n}(G)=f_{n}(B)$ (see, for instance, [12, v. I, section 34, p. 170 and p. 173, Exercise 2]). But $B G^{p^{\omega}} / G^{p^{\omega}} \cong B$, therefore $f_{n}\left(G / G^{p^{\omega}}\right)=f_{n}(G)$ whenever $n \geq 0$. Consequently, if $A$ is a Prüfer abelian $p$-group, then $f_{\alpha}(A)=1$ for each $\alpha \leq \omega$ and $f_{\alpha}(A)=0$ for each $\alpha>\omega$.

Received November 5, 2008; revised June 25, 2009.

2000 Mathematics Subject Classification. 16S34, 16U60, 20K10, 20K20, 20K21.

Key words and phrases. Prüfer groups, cyclic groups, direct sums of cyclic groups, units, UlmKaplansky invariants, direct factors. 
From [19, Theorem 6], an abelian $p$-group $A$ such that $A^{p^{\omega}}$ is countable and $A / A^{p^{\omega}}$ is a direct sum of cyclic groups can be decomposed as $C \times D$, where $C$ is countable and $D$ is a direct sum of cyclic groups. In the case of Prüfer groups we observe that $D=1$.

In the theory of commutative group algebras there exists a long-standing conjecture (see, e.g., [1]) stating that $S(R G) / G$ is a direct factor of a direct sum of generalized Prüfer groups, defined as in [12, v. II, section 81, pp.103-104], whenever $G$ is a $p$-group and $R$ is a perfect ring with characteristic $p$.

Under this point of view, we are motivated to give a criterion in order to check whether $S(R G)$ and $S(K G)$ are Prüfer groups. The same purpose we pursue for $S(R G) / G$ and $S(K G) / G$, provided $G$ is a $p$-group. The main results of this article show that none of $S(R G) / G$ and $S(K G) / G$ cannot be Prüfer's groups, against ignoring "a direct sum" in the above conjecture. For some positive solutions of this conjecture the reader can see [1], [9], [14], [17] and [18].

\section{Main results}

The present paper extends [2, Section D]. The theorems are distributed into two sections.

\section{Commutative modular group algebras and Prüfer groups}

The next two lemmas are straightforward and, therefore, their proofs are omitted.

Lemma 1. If $g$ is an element of finite order of $G$, then

$$
|\langle g\rangle|=o(g) \text {. }
$$

where $\langle g\rangle$ is a finite cyclic group and $o(g)$ is the order of $g$.

Lemma 2. (a) Assume $G$ is p-primary, and $k \in \mathbb{N}$. Then $S^{p^{k}}(R G)=1$ if and only if $G^{p^{k}}=1$.

(b) Assume $R$ has no nilpotent elements and $k \in \mathbb{N}$. Then $S^{p^{k}}(R G)=1$ if and only if $G_{p}^{p^{k}}=1$.

Lemma 3.([3, p. 8]) We have $S(R G)=G_{p}$ if and only if one of the following conditions is true:

(i) $G=1$;

(ii) $G \neq G_{p}=1$ and $N(R)=0$;

(iii) $|G|=2$ and $|R|=2$.

Proposition 1. $V(R G)$ is a cyclic p-group if and only if one of the following equalities holds:

(1) $G=1$; 
(2) $p=|G|=|R|=2$.

Proof. Obviously, if $V(R G)$ is cyclic, then $G$ is cyclic. From Lemmas 1 and 2(a), we deduce $|V(R G)|=|G|$ and so $V(R G)=G$. Lemma 3 concludes the necessity.

Conversely, the result follows again by Lemma 3 .

We shall now generalize Proposition 1.

Proposition 2. $S(R G)$ is cyclic if and only if one of the following is valid:

(1) $G=1$;

(2) $G \neq G_{p}=1$ and $N(R)=0$;

(3) $|G|=2$ and $|R|=2$;

(4) $G_{p}=1,|G|=2$ and $|N(R)|=p \geq 3$.

Proof. First, assume that $S(R G)$ is cyclic. We distinguish two cases, namely:

Case 1: $N\left(R^{p^{i}}\right)=N^{p^{i}}(R) \neq 0$ for all $i \in \mathbb{N}$.

(a) Since there is $g \in G$ with $g^{n} \neq 1$ for every $n \in \mathbb{N}, G$ is either torsion-free or mixed. Let $0 \neq r \in N(R)$. For $m \in \mathbb{N}$ we construct the infinite number of different elements $1+r\left(1-g^{p^{m}}\right) \in S(R\langle g\rangle)$ where $S(R\langle g\rangle)$ is cyclic, hence finite, as a subgroup of $S(R G)$. Note that $\langle g\rangle$ is an infinite cyclic group in this situation. Therefore, $S(R\langle g\rangle)=1$, i.e., $g=1$ which is a contradiction.

(b) By what we have just shown in the previous point, $G$ must be torsion.

(b.1) $N\left(R^{p^{i^{\prime}}}\right)=N\left(R^{p^{i^{\prime}+1}}\right)$ for some $i^{\prime} \in \mathbb{N}_{0}=\mathbb{N} \cup\{0\}$. Thus $1+I\left(N\left(R^{p^{i^{\prime}}}\right) G_{q} ; G_{q}\right)=$ 1 being both a divisible and cyclic group since $G_{q}$ is $p$-divisible whenever $q \neq p$ is a prime. Therefore, $G_{q}=1$, i.e., $G$ is $p$-primary.

(b.2) $N\left(R^{p^{i}}\right) \neq N\left(R^{p^{i+1}}\right)$ for each $i \in \mathbb{N}_{0}$. Then the sequence $N(R), N\left(R^{p}\right)$, $N\left(R^{p^{2}}\right), \ldots, N\left(R^{p^{i}}\right), \ldots$ has an infinite number of different members. Choose the elements $x_{i}=1+r_{i}^{p^{i}}\left(1-g_{q}\right)$ where $r_{i} \in N(R) ; r_{i}^{p^{i}} \in N\left(R^{p^{i}}\right)$ with $r_{i}^{p^{i}} \neq r_{i+1}^{p^{i+1}}$ and $g_{q} \in G_{q}$. Thus $x_{i} \neq x_{i+1}$ and $S\left(R G_{q}\right)$ is infinite cyclic, i.e., $S\left(R G_{q}\right)=1$, whence $G_{q}=1$. Finally, we conclude that $G$ is $p$-torsion.

In that aspect Proposition 1 substantiates our claim.

Case 2: $N\left(R^{p^{j}}\right)=0$ for some $j \in \mathbb{N}_{0}$.

If $P$ is a commutative ring with unity of characteristic $p$ such that $N(P)=0$, as in Proposition 1, $S(P G)$ cyclic yields $S(P G)=G_{p}$, because we have $\exp (S(P G))=\exp \left(G_{p}\right)$ by Lemma 2(b).

Consequently, $S(R G)$ cyclic gives $S\left(R^{p^{j}} G\right)$ is cyclic, hence in view of the conclusions above, $S\left(R^{p^{j}} G\right)=G_{p}$. So, Lemma 3 leads us to $G=1$, or $G \neq G_{p}=1$, or $G=G_{p} \neq 1$, $\left|R^{p^{j}}\right|=2,|G|=2$. We will consider the following two subcases. 
(2.1) Assume $G_{p} \neq 1$. Hence $R$ is a field which follows from Proposition 1 since $G$ is a $p$-group.

We also note that $R^{p^{j}}$ is a field and $N(R)=0$, whence $R$ is a field. If $0 \neq r \in R$, then $0 \neq r^{p^{j}} \in R^{p^{j}}$. So, there exists some $0 \neq \alpha \in R^{p^{j}}$ such that $r^{p^{j}} \cdot \alpha=r\left(r^{p^{j}-1} \cdot \alpha\right)=1$. Therefore, $r$ is invertible in $R$ and this allows us to deduce that $R$ is a field.

(2.2) Assume $G_{p}=1$ and $N(R) \neq 0$. Each element of $S(R G)$ will be of the form $1+r_{1} g_{1}+\ldots+r_{k} g_{k}$, where $0 \neq r_{i} \in N(R)$ with $\sum_{i=1}^{k} r_{i}=0$ and $g_{i} \in G ; 1 \leq i \leq k$. Clearly, $|N(R)| \leq|S(R G)|$ and $|G| \leq|S(R G)|$ since $1+r(1-g)=1+r^{\prime}\left(1-g^{\prime}\right)$ if and only if $r=r^{\prime}$ and $g=g^{\prime}$, where $r, r^{\prime} \in N(R) \backslash\{0\}$ and $g, g^{\prime} \in G \backslash\{1\}$. Since $\operatorname{tr} \neq 0$ whenever $1 \leq t \leq p-1$, we obtain that $|N(R)| \geq p$. On the other hand, if $|G| \geq 3$, then as above $|N(R)|<|S(R G)|$ whenever $|S(\bar{R} G)|<\aleph_{0}$.

Now consider $S(R G)=\left\{1, v, v^{2}, \ldots, v^{p^{m}-1} \mid v^{p^{m}}=1\right\}$, where $1 \leq m \leq j$; thus $|S(R G)|=p^{m}$. Moreover, $|R| \geq 4,|N(R)| \leq p^{m}$ and $|G|<p^{m}$; we observe that $|G|=p^{m}$ means $G=G_{p}=1$.

Suppose $j=1$ (hence $m=1$ ). But $|S(R G)|=p$ and $p \geq 3$, since in the remaining case when $p=2$ we deduce $|G|<2$, i.e., $G=1$. Because of the above given inequalities, we obtain $|N(R)|=p$ so $N(R)=\left\{0, r, 2 r, \ldots,(p-1) r \mid r^{2}=0\right\}$ and $N^{p}(R)=N\left(R^{p}\right)=0$. Utilizing the above ideas, it follows that $|G|=2$.

Now, let $m \geq 2$, so $j \geq 2$. Since $S\left(R^{p^{j-1}} G\right) \subseteq S(R G)$ is cyclic and $S^{p}\left(R^{p^{j-1}} G\right)=$ 1, we conclude that $\left|S\left(R^{p^{j-1}} G\right)\right|=p$. Hereafter, the above step can be successfully employed to deduce that $|G|=2$; of course $N\left(R^{p^{j-1}}\right) \neq 0$, otherwise $1 \leq m \leq j-1$ and the first point will be started for $j=2$. That is why, $|N(R)|=p^{m}$ and $\left|N\left(R^{p^{j-1}}\right)\right|=p$.

Evidently, every element of $S(R G)$ is of the form $1+r(1-g)$, where $r \in N(R)$. If $r \in N(R)$, it follows that $\{0, r, 2 r, \ldots,(p-1) r\} \subseteq N(R)$. Likewise, if $r \in N(R)$ with $r^{s}=0, s \geq 2$ and $r^{s-1} \neq 0$, it holds that the set $\left\{r, r^{2}, \ldots, r^{s-1}\right\}$ of $s-1$ elements is a set of different nilpotent elements. All of these arguments lead us to the evaluation that $|N(R)| \geq 1+(s-1)(p-1)$ for $s \geq 2$. Since the sum of nilpotent elements is a nilpotent, there are $s-2$ nilpotent elements by considering the different sums $r+r^{2}, \ldots, r+r^{s-1}$. Finally, we compute that $|N(R)| \geq(s-1)(p-1)+1+s-2=(s-1) p, s \geq 2$; of course there is an exact estimation of the cardinality of the finite ideal $N(R)$, but however this inequality works.

So, if $S(R G)$ is cyclic of $\exp (S(R G))=p^{m}$ for $m \geq 2$, it is routine to verify that there exists a nilpotent element $\beta \in R$ such that $\beta^{p^{m}}=0, \beta^{p^{m}-1} \neq 0$ for $m \geq 2$ and $S(R G)=\langle 1+\beta(1-g)\rangle$.

By what we have just shown for $s=p^{m}$ with $m \geq 2$, it follows that $|N(R)| \geq$ $\left(p^{m}-1\right) p>p^{m}$ whenever $p \geq 2, m \geq 2$; notice that $p^{m}-1>p^{m-1}$ holds even for $p \geq 3$, $m \geq 1$. This contradicts our equality $|N(R)|=p^{m}$.

Finally, we find that $m<2$ and $j<2$ when $S(R G)$ is cyclic. The result follows.

Conversely, assume that conditions (1) - (4) are satisfied. For points (1)-(3) we directly apply Lemma 3 to conclude that $S(R G)=G_{p}$ and since $G_{p}$ is cyclic, the result follows. If now (4) holds, it is easily verified that every element of $S(R G)$ is of the type $1+n r(1-g)=(1+r(1-g))^{n}$, where $0 \leq n \leq p-1$ and $r \in N(R)$ with $r^{2}=0$. So, $S(R G)$ is a cyclic group of order $p$. 
Remark 1. $1 \neq S(R G)$ cyclic yields that $N(R)$ is finite. On the other hand, $1 \neq S(R G)$ cyclic implies that $R$ is perfect provided $G_{p} \neq 1$. From [7, Example 9], $N(R)=0$. Actually, Cases 1 and 2 in Proposition 2 may be reduced to $N(R)=0$ and $G_{p} \neq 1$; or $N(R) \neq 0$ and $G_{p}=1$, or $N(R) \neq 0$ and $G_{p} \neq 1$.

The reader can see also [4, Theorem, pp.262-263] where we have established a criterion for $S(R G)$ to be a direct sum of cyclic groups of the same order $p^{t}$ for $t \geq 1$.

The next constructions illustrate Proposition 2.

Example 1. Consider the following rings and their nil-radicals.

(1) $R=\{0,1,-1\}$, char $(R)=3, N(R)=\{0\}$;

(2) $R=\left\{0,1, r, 1-r \mid r^{2}=0\right\}$, $\operatorname{char}(R)=2, N(R)=\{0, r\}$;

(3) $R=\left\{0,1,-1, r, 2 r, r+1, r-1,-r-1,1-r \mid r^{2}=0\right\}$, $\operatorname{char}(R)=3, N(R)=\{0, r, 2 r\}$;

(4) $N(R)=\left\{0, r, r^{2}, r+r^{2} \mid r^{3}=0\right\}, \operatorname{char}(R)=2$;

(5) $N(R)=\left\{0, r, 2 r, r^{2}, 2 r^{2}, r+r^{2},-r-r^{2}, r-r^{2}, r^{2}-r \mid r^{3}=0\right\}, \operatorname{char}(R)=3$.

Suppose now that $|S(R G)|=4(p=2, m=2)$, that $|G|=3$ with $G=\langle g\rangle$ and that $R$ is as in 2). Therefore, $S(R G)=\left\{1,1+r(1-g), 1+r\left(1-g^{2}\right), 1+r g(1-g)\right\}$. Because $S^{2}(R G)=1, S(R G)$ is not cyclic but is a direct sum of the two cyclic groups $\{1,1+r(1-g)\}$ and $\left\{1,1+r\left(1-g^{2}\right)\right\}$ each of which is with order 2 ; we observe that $(1+r(1-g))\left(1+r\left(1-g^{2}\right)\right)=1+r g(1-g)$.

Let now $p=3$ and $m=2$, as well as $|G|=2$ and $N(R)$ be as in 5). Consequently, $S(R G)=\{1+\alpha(1-g) \mid \alpha \in N(R)\}$ is of power 9. But $S(R G)$ is not cyclic of order 9 since $S^{p}(R G)=1$, i.e., $(1+\alpha(1-g))^{3}=1+\alpha^{3}\left(1-g^{3}\right)=1$; note that $N^{p}(R)=0$.

Proposition 3. Suppose that $G$ is a p-group. Then $V(R G) / G$ is cyclic if and only if one of the following conditions is true:

(1) $G=1$;

(2) $p=|R|=|G|=2$;

(3) $p=|G|=2$ and $|R|=4$;

(4) $p=|R|=2, G^{2} \neq 1$ and $|G|=4$;

(5) $p=|R|=|G|=3$.

Proof. Let $V(R G) / G$ be a nontrivial cyclic group of order $p^{m}$ for $m \geq 1$. So, $\bar{V}=$ $V(R G) / G=\left\{\overline{1}, \bar{v}, \bar{v}^{2}, \ldots, \bar{v}^{p^{m}-1} \mid \bar{v}^{p^{m}}=1\right\}$ for some element $\bar{v}=\left(r_{1} g_{1}+\ldots+r_{n} g_{n}\right) G$; $0 \neq r_{i} \in R$ with $\sum_{i=1}^{n} r_{i}=1, g_{i} \in G ; 1 \leq i \leq n$. If $G$ is decomposable, then by [1, Theorem (Direct Factor)], $V(R G) / G$ must be decomposable (see also [9]). But this is impossible and thus $G$ is indecomposable.

Since $(V(R G) / G)^{p^{m}}=\overline{1}$, we have $V^{p^{m}}(R G) G=G$, i.e., $V\left(R^{p^{m}} G^{p^{m}}\right)=G^{p^{m}}$. Using Lemma 3, we derive $G^{p^{m}}=1$, or $G^{p^{m}} \neq 1, p=\left|R^{p^{m}}\right|=\left|G^{p^{m}}\right|=2$. Both $R$ and $G$ are finite. Moreover either $G^{p^{k}} \neq 1$ and $\left|R^{p^{k}}\right|>2$, or $\left|G^{p^{k}}\right|>2$, for $0 \leq k<m$. 
We shall distinguish five cases:

Case 1: $p=2, m=1$. Thus $V(R G) / G=\left\{\overline{1}, \bar{v} \mid \bar{v}^{2}=1\right\}$. Assume that $|R|>2$. Hence, $\overline{1} \neq[1+r(1-g)] G=\bar{v}$ for $0,1 \neq r \in R$ and $1 \neq g \in G$ with $[1+r(1-g)]^{2} \in G$, i.e., $1+r^{2}\left(1-g^{2}\right) \in G$. This is equivalent to $r^{2}=0$ or $r^{2}=1$ or $g^{2}=1$. Besides, we consider $\left[1+r^{\prime}(1-h)\right] G$ for some $r^{\prime} \in R \backslash\{0,1, r\}$ and $h \in G \backslash\{1, g\}$ such that $\left[1+r^{\prime}(1-h)\right] G=$ $[1+r(1-g)] G$, i.e., $r^{\prime}=1+r$ and $h=g^{-1}$. Consequently, $[1+r(1-g)] G \neq\left[1+r\left(1-g^{-1}\right)\right] G$ when $g \neq g^{-1}$. That is why, $|G|=2$ and $|R|=4$.

Let us now $|R|=2$, whence $|G|>2$ and more precisely $|G| \geq 4$. This will be studied in the next case.

Case 2: $p=2, m \geq 2$. Starting with $m=2, V(L G) / G \subseteq V(R G) / G$ is cyclic of order 2 or 4 where $L=\{0,1\} \leq R$. Since $G^{4}=1$ and $G^{2} \neq 1$, or $G^{4} \neq 1$ with $\left|R^{4}\right|=\left|G^{4}\right|=2$, there exists $1 \neq g \in G$ such that $g^{2} \neq 1, g^{3} \neq 1$ whence $\left\{1, g, g^{2}, g^{3} \mid g^{4}=1\right\} \leq G$.

First, $|V(L G) / G|=2$, and $|G|=4$, i.e., $G=\left\{1, g, g^{2}, g^{3} \mid g^{4}=1\right\}$, so $G^{2}=\left\{1, g^{2}\right\}$. Since $V(R G)=G \cup\left\{1+g+g^{2}, 1+g+g^{3}, 1+g^{2}+g^{3}, g+g^{2}+g^{3}\right\}$, it is immediate that $\bar{V}=\left\{\overline{1},\left[1+g+g^{2}\right] G\right\}$.

If now $|G|=8$, i.e., $G=\left\{1, g, \ldots, g^{7} \mid g^{8}=1\right\}$, we observe that $\bar{V}$ contains five different elements, that are $\overline{1},\left(1+g+g^{2}\right) G,\left(1+g+g^{3}\right) G,\left(1+g+g^{2}+g^{3}+g^{4}\right) G$ and $\left(1+g+g^{2}+g^{3}+g^{4}+g^{5}+g^{6}\right) G$. This contradicts the power of $\bar{V}$ which is precisely 4 .

If $|G|=4$ and $|R|=4$, then $G=\left\{1, g, g^{2}, g^{3} \mid g^{4}=1\right\}$ and $R=\{0,1, r, 1+r\}$. Furthermore, we see that $\bar{V}=\left\{\overline{1},\left(1+g+g^{2}\right) G,(1+r-r g) G,\left(1+r-r g^{2}\right) G,\left(1+r-r g^{3}\right) G\right\}$ consists of five different elements because $1+r \neq-r$, but this is not true. Thus $|R|<4$ or $|G|<4$, i.e., $|R| \leq 2$ or $|G| \leq 2$.

Next, for $m \geq 3$ we observe that $V\left(L^{p^{m-2}} G^{p^{m-2}}\right) G / G \cong V\left(L^{p^{m-2}} G^{p^{m-2}}\right) / G^{p^{m-2}}$ is a cyclic group of order $p^{2}$ as a subgroup of $V(L G) / G$. This is exactly the previous step. Thus, when $m \geq 2, V(R G) / G$ is not cyclic.

Case 3: $p=3, m=1$. Since $p=3$ there is $r \in R$ with $r \neq 0,1$, hence $\{0,1,-1\} \subseteq R$. Moreover, $G^{3}=1$ and $G^{2} \neq 1$. Let us now $|R|=3$, i.e., $R=\{0,1,-1\}$ and $|G|=3$, that is, $G=\left\{1, g, g^{2} \mid g^{3}=1\right\}$. Therefore, $V(R G)=\{1,1+g(1-g), 1-g(1-g),-g-$ $\left.g^{2},-1-g, g, g^{2}\right\}$ and $\bar{V}=\{\overline{1},[1+g(1-g)] G,[-1-g] G\}$. Moreover, we calculate that $[1+g(1-g)] G=\left[1+g-g^{2}\right] G=\left[1-g+g^{2}\right] G=\left[1+2 g+g^{2}\right] G=[-1-g]^{2} G=[(-1-g) G]^{2}$.

If we suppose that $|R|>3$ or $|G|>3$, i.e., $|R| \geq 9$ or $|G| \geq 9$, it is not difficult to obtain in the same manner that $|\bar{V}|>3$, which is a contradiction.

Case 4: $p=3, m \geq 2$. Start with $m=2$. Certainly, $G^{9}=1$ and so $|G|=9$ since otherwise if $|G|=3$ it follows that $G^{3}=1$, a contradiction. Thus $G=\left\{1, g, g^{2}, \ldots, g^{8} \mid g^{9}=1\right\}$ and as above $R \supseteq\{0,1,-1\}$. Consider the elements $\left(1+g-g^{k}\right) G$ for $2 \leq k \leq 8$. It is only a technical matter to check that $\left(1+g-g^{k}\right) G \neq\left(1+g-g^{j}\right) G$ whenever $g^{k} \neq g^{j}$ and $2 \leq j \leq 8$, because $1 \neq-1$. Moreover, two different elements are also $\left(1+g+g^{2}+g^{3}\right) G$ and $\left(1+g+g^{2}-g^{3}-g^{4}\right) G$. A crucial approach here is that the canonical forms of these elements are with different lengths. Consequently, $\bar{V} \supseteq\left\{\overline{1},\left(1+g-g^{k}\right) G\right.$ for $2 \leq k \leq 8$, $\left.\left(1+g+g^{2}+g^{3}\right) G,\left(1+g+g^{2}-g^{3}-g^{4}\right) G\right\}$ contains ten elements. This gives a contradiction and finishes the step $m=2$. 
When $m \geq 3$ we have $|G| \geq 27$ and, therefore, we can copy the idea from Case 2 .

Case 5: $p \geq 5$. Begin with $m=1$. Since the characteristic of $R$ is $p$ and $R \supseteq$ $\{0,1,2, \ldots, p-1\}$, it holds that $|R| \geq p$. Moreover, $G^{p}=1$ and $G^{s} \neq 1$ for $1 \leq s \leq p-1$. It is a routine technical exercise to verify that $\left(1+\left(1-g^{k}\right)\right) G=\left(2-g^{k}\right) G \neq\left(2-g^{j}\right) G=$ $\left(1+\left(1-g^{j}\right)\right) G$ when $g^{k} \neq g^{j}$ and $1 \leq k \neq j \leq p-1$ for some $g \in G$. In this way $(1+g(1-g)) G \neq\left(2-g^{k}\right) G$ for all $1 \leq k \leq p-1$. Finally, $\bar{V}$ contains the set of $p+1$ different elements $\left\{1,(2-g) G,\left(2-g^{2}\right) G, \ldots,\left(2-g^{p-1}\right) G,(1+g(1-g)) G\right\}$ while $|\bar{V}|=p$. This contradiction shows that this case cannot happen. After this, because $V\left(R^{p^{m-1}} G^{p^{m-1}}\right) / G^{p^{m-1}} \cong V\left(R^{p^{m-1}} G^{p^{m-1}}\right) G / G \subseteq V(R G) / G$ is cyclic of order $p$ whenever $m \geq 2$, we conclude that the case is contradictory. This completes the necessity.

As for the sufficiency, we observe that for the first four situations we have $|V(R G) / G|=$ 1 , hence $V(R G) / G=\overline{1}$, or $|V(R G) / G|=2$. The fifth dependence was considered in Case 3 above.

Example 2. There are four special commutative unitary rings of power 4 and with characteristic 2 which illustrate the criteria in Propositions 2 and 3. Specifically, they are the following:

(1) $R=\left\{0,1, r, 1+r \mid r^{2}=0\right\}, N(R)=\{0, r\}$;

(2) $R=\left\{0,1, r, 1+r \mid r^{2}=1\right\}, N(R)=\{0,1+r\}$;

(3) $R=\left\{0,1, r, 1+r \mid r^{2}=r\right\}, N(R)=\{0\}$ and $R$ has two zero divisors $\{r, 1+r\}$ which are idempotents, so $R$ is perfect;

(4) $R=\left\{0,1, r, 1+r \mid r^{2}=1+r\right\}, N(R)=\{0\}$ and $R$ has three units $\{1, r, 1+r\}$, i.e., $R$ is a perfect field.

We are now prepared to proceed by proving the main assertions. In the next two theorems we use results on Ulm-Kaplansky invariants of $V(R G) / G$ provided $G$ is a $p$-group and $R$ is a perfect ring of prime characteristic $p$ (see details in [10, p. 138], Theorem 6 and p. 141, Remark]). Utilizing the same ideas, it easily follows that these Ulm-Kaplansky invariants are either infinite or zero when $G$ is infinite and $R$ is not necessarily perfect.

Theorem 1. Suppose $G$ is a p-group or $R$ is a ring with no nilpotent elements. Then $S(R G)$ cannot be a Prüfer group.

Proof. (1) Assume $G=G_{p}$ and by contradiction, let $S(R G)=V(R G)$ be a Prüfer group. Thus $V^{p^{\omega}}(R G)=V\left(R^{p^{\omega}} G^{p^{\omega}}\right)$ is cyclic of order $p$, hence Proposition 1 and its proof guarantee that $V\left(R^{p^{\omega}} G^{p^{\omega}}\right)=G^{p^{\omega}}$. On the other hand, $V(R G)$ is countable and so $V(R G) / G$ is countable. Since $G$ is nice in $V(R G)$ (cf., [10, p.135, Lemma 1]), we deduce that $(V(R G) / G)^{p^{\omega}}=V^{p^{\omega}}(R G) G / G=\overline{1}$, whence $V(R G) / G$ is separable. By the second Prüfer's theorem (see [12, v. I, Theorem 17.3]), $V(R G) / G$ is a direct sum of cyclic groups. Furthermore, because of the purity of $G$ in $V(R G)$, a result due to L. Kulikov (e.g., [12, 
v. I, Theorem 28.2]) is applicable to obtain $V(R G) \cong G \times(V(R G) / G)$. Therefore, $V(R G) / G^{p^{\omega}} \cong\left(G / G^{p^{\omega}}\right) \times(V(R G) / G)$. Assume $G=G^{p^{\omega}}$. Then $G$ is both cyclic and divisible. This gives a contradiction when $G \neq 1$. Thus we conclude that $G \neq G^{p^{\omega}}$, whence $G$ is infinite because $G^{p^{\omega}} \neq 1$. Moreover, $V(R G) / G^{p^{\omega}}=V(R G) / V^{p^{\omega}}(R G)$ has Ulm-Kaplansky functions equal to 1 . On the other hand, conforming with $[12$, v. I, section 37, p.185, Exercise 8], these invariants for $V(R G) / G^{p^{\omega}}$ are equal to the sum of the Ulm-Kaplansky invariants of $G / G^{p^{\omega}}$ and $V(R G) / G$ respectively. Moreover, [10, p.138, Theorem 6] applies to show that $V(R G) / G$ has infinite Ulm-Kaplansky invariants when either $G$ or $R$ is infinite and $G \neq G^{p}$; as early observed $G \neq G^{p}$ holds. If both $G$ and $R$ are finite, then $S(R G)$ is obviously finite whence it is not Prüfer. Consequently, $V(R G)=G$, so Lemma 3 leads us to $|R|=|G|=2$, which is the desired contradiction with the infinite cardinality of the Prüfer groups.

(2) Assume $N(R)=0$. This case can be processed similarly as that in (1).

Theorem 2. Suppose $G$ is a p-group and $R$ is a commutative unitary ring of prime characteristic $p$. Then $V(R G) / G$ cannot be a Prüfer group.

Proof. Assume the contrary. In view of the definition and our assumption $(V(R G) / G)^{p^{\omega}}=$ $V^{p^{\omega}}(R G) G / G=V\left(R^{p^{\omega}} G^{p^{\omega}}\right) G / G \cong V\left(R^{p^{\omega}} G^{p^{\omega}}\right) / G^{p^{\omega}}$ is cyclic of order $p$, whence $G^{p^{\omega}} \neq$ $V\left(R^{p^{\omega}} G^{p^{\omega}}\right)$ and thus $G$ is infinite. However, by the above commentaries, $V(R G) / G$ should be with Ulm-Kaplansky functions precisely 1. But, complying with [10, p.138, Theorem 6], when $G$ is infinite we deduce that these invariants computed for $V(R G) / G$ are infinite or 0 . So, we obtain the wanted contradiction.

In case $G$ is finite, we yield that $V(R G) / G$ is bounded whence it is not a Prüfer group.

The following illustrates Theorem 1.

Example 3. Consider $V(R G)=\oplus_{n<\omega} \mathrm{Z}\left(p^{n}\right)$. Evidently, $|V(R G)|=\aleph_{0} \Longleftrightarrow|R|+$ $|G|=\aleph_{0}$ with $|R| \leq \aleph_{0}$ and $|G| \leq \aleph_{0}$. Besides, $V^{p^{\omega}}(R G)=1 \Longleftrightarrow G^{p^{\omega}}=1$ and, for each $n \geq 1, V^{p^{n}}(R G) \neq 1 \Longleftrightarrow G^{p^{n}} \neq 1$. By assumption, for every $k \geq 0$, the $k$-Ulm-Kaplansky invariants of $V(R G)$ are 1 , while owing to [22, Theorem 7] they are equal to 0 or to $\max \left(\left|R^{p^{k}}\right|,\left|G^{p^{k}}\right|\right)$ if either $\left|R^{p^{k}}\right| \geq \aleph_{0}$ or $\left|G^{p^{k}}\right| \geq \aleph_{0}$. In the case $k=0$, we obtain a contradiction. That is why, $V(R G) \neq \oplus_{n<\omega} \mathrm{Z}\left(p^{n}\right)$.

Another idea to show that the equality $V(R G)=\oplus_{n<\omega} \mathrm{Z}\left(p^{n}\right)$ is not true is like this: If yes, $G$ should be a direct sum of cyclic groups, hence, in virtue of [5] or [6], so is $V(R G) / G$. Furthermore, as we have just seen above, $V(R G)=G \times V(R G) / G$. But then the Ulm-Kaplansky functions argument means that $V(R G)=G$, i.e., by Lemma 3 , $V(R G)$ is finite which is against our hypothesis. 
Commutative semisimple group algebras and Prüfer groups

Theorem 3. Suppose $G$ is a p-group and $K$ is the first kind field with respect to $p$. Then $S(K G)$ cannot be a Prüfer group.

Proof. Let $S(K G)$ be a Prüfer group. By definition, $S^{p^{\omega}}(K G)$ is cyclic of order $p$. Exploiting [20, Theorem 19], $S^{p^{\omega}}(K G)$ is divisible. Thus $S^{p^{\omega}}(K G)=1$, a contradiction.

Note 1: In the situation of Theorem 3, $S(K G) / S^{p^{\omega}}(K G) \cong S(K G)$ has Ulm-Kaplansky invariants equal to 1 . Taking into account [21, Theorem 7], $S(K G)$ possesses UlmKaplansky functions equal to 0 or to $|B|$ where $B$ is the basic subgroup of $G$. Hence, $B=1$, i.e., $G$ is divisible. Furthermore, in virtue of [8, Theorem 4], we derive that $S(K G)$ is divisible. But it is reduced, i.e., $S(K G)=1$, a contradiction.

Theorem 4. Suppose $G$ is a p-group and $K$ is a field of the first kind with respect to $p$. Then $S(K G) / G$ cannot be a Prüfer group.

Proof. According to [11, Proposition 1], $(S(K G) / G)^{p^{\omega}}$ is always divisible (see also [8]). So, it cannot be a cyclic group of order $p$. That is why $S(K G) / G$ cannot be a Prüfer group, as asserted.

Note 2: As in Note 1, the Ulm-Kaplansky arguments from [11] are also applicable to deduce that $S(K G) / G$ cannot be, in fact, a Prüfer group.

Theorem 5. Suppose $G$ is a p-group and $K$ is the second kind field with respect to $p$. Then $S(K G)$ cannot be a Prüfer group.

Proof. Owing to [20, p.36 and Theorem 21], we find that $S(K G)$ is a direct sum of co-cyclic groups, hence it is not a Prüfer group.

Theorem 6. Suppose $G$ is a p-group and $K$ is a field of the second kind with respect to p. Then $S(K G) / G$ cannot be a Prüfer group.

Proof. If $G$ is finite, $S(K G)$ is finite or divisible whence so is $S(K G) / G$. Therefore, it is not a Prüfer group.

When $G$ is infinite, we employ [20, p.45, Theorem 21] to infer that $S(K G) / G$ need not be a Prüfer group. In fact, if $p \neq 2$, then $S(K G)$ is divisible, whereas if $p=2$ and $G^{p} \neq 1$, then $S^{p}(K G)$ is divisible. Thus in both cases $(S(K G) / G)^{p}=S^{p}(K G) G / G$ is divisible, and consequently $(S(K G) / G)^{p^{\omega}}=(S(K G) / G)^{p}$ is not cyclic. When $G^{p}=1$, we observe that $S(K G)$ is bounded by $p$, whence the same is $S(K G) / G$.

So, in any event, $S(K G) / G$ is not a Prüfer group, as expected.

Example 4. As in the modular case, one can illustrate in Theorem 3 that the equality $S(K G)=\oplus_{n<\omega} \mathrm{Z}\left(p^{n}\right)$ is not valid by applying [20] and [21]; see the proof of Theorem 3 as well. 


\section{Global case}

Combining both the modular and semi-simple cases, we establish the following.

Global Theorem 7. Let $G$ be a p-group and let $F$ be a field of arbitrary characteristic. Then $S(F G)$ and $S(F G) / G$ cannot be Prüfer groups.

Proof. Each field has characteristic $p$ or characteristic different from $p$. These fields with characteristic $\neq p$ are either of the first kind with respect to $p$ or of the second kind with respect to $p$, respectively. Henceforth, the foregoing theorems work.

\section{Acknowledgement}

The author would like to express his warm thanks to the specialist referee for the critical remarks which considerably improved on the original manuscript.

\section{References}

[1] P. V. Danchev, Modular group algebras of coproducts of countable abelian groups, Hokkaido Math. J. 29 (2000), 255-262.

[2] -, Sylow p-subgroups of modular abelian group rings, Compt. rend. Acad. bulg. Sci. (2) 54 (2001), 5-8.

[3] -, Completely characteristic and large subgroups in commutative group rings, Compt. rend. Acad. bulg. Sci. (8) 54 (2001), 5-8.

[4] - , Homogeneous primary components in abelian group rings, Math. Balkanica 15 (2001), 261-264.

[5] —, Normed units in abelian group rings, Glasgow Math. J. 43 (2001), 365-373.

[6] - Invariants for group algebras of splitting abelian groups with simply presented components, Compt. rend. Acad. bulg. Sci. 55 (2002), 5-8.

[7] —, Basic subgroups in abelian group rings, Czechoslovak Math. J. 52 (2002), 129-140.

[8] —, Sylow p-subgroups of abelian group rings, Serdica Math. J. 29 (2003), 33-44.

[9] - Commutative group algebras of direct sums of countable abelian groups, Kyungpook Math. J. 44 (2004), 21-29.

[10] 一, Ulm-Kaplansky invariants for $S(R G) / G_{p}$, Bull. Inst. Math. Acad. Sinica 32 (2004), $133-144$

[11] —, Ulm-Kaplansky invariants of $S(K G) / G$, Bull. Polish Acad. Sci. - Math. 53 (2005), $147-156$

[12] L. Fuchs, Infinite Abelian Groups, volumes I and II, Academic Press, New York, 1970 and 1973.

[13] P. D. Hill, On primary groups with uncountable many elements of infinite height, Arch. Math. Basel 19 (1968), 279-283.

[14] P. D. Hill and W. D. Ullery, On commutative group algebras of mixed groups, Comm. Algebra 25 (1997), 4029-4038.

[15] M. I. Kargapolov and J. I. Merzljakov, Fundamentals of the Theory of Groups, Springer, New York, 1979. 
[16] A. G. Kurosh, The Theory of Groups, Chelsea Publ. Co., New York, 1960.

[17] W. L. May, Modular group algebras of totally projective p-primary groups, Proc. Amer. Math. Soc. 76 (1979), 31-34.

[18] W. L. May, Modular group algebras of simply presented abelian groups, Proc. Amer. Math. Soc. 104 (1988), 403-409.

[19] C. K. Megibben, On high subgroups, Pac. J. Math. 14 (1964), 1353-1358.

[20] T. Zh. Mollov, Sylow p-subgroups of the group of normed units of semisimple group algebras of uncountable abelian p-groups, Pliska Stud. Math. Bulgar. 8 (1986), 34-46. (In Russian.)

[21] -, Ulm-Kaplansky invariants of the Sylow p-subgroups of the group of normed units of semisimple group algebras of infinite separable abelian p-groups, Pliska Stud. Math. Bulgar. 8 (1986), 101-106. (In Russian.)

[22] T. Zh. Mollov and N. A. Nachev, Ulm-Kaplansky invariants of the group of normalized units of modular group rings of primary abelian groups, Serdica 6 (1980), 258-263. (In Russian.)

13, General Kutuzov Street, block 7, floor 2, flat 4, 4003 Plovdiv, Bulgaria.

E-mail: pvdanchev@yahoo.com

13, General Kutuzov Street, block 7, floor 2, flat 4, 4003 Plovdiv, Bulgaria.

E-mail: pvdanchev@mail.bg 